\title{
CONSULTA DE ENFERMAGEM À SAÚDE DE HOMENS NA ATENÇÃO PRIMÁRIA À SAÚDE: ESTRATÉGIAS E DESAFIOS
}

\section{NURSING CONSULTATION FOR MEN'S HEALTH IN PRIMARY HEALTH CARE: STRATEGIES AND CHALLENGES}

\section{CONSULTA DE ENFERMERÍA PARA LA SALUD DEL HOMBRE EN LA ATENCIÓN PRIMARIA DE LA SALUD: ESTRATEGIAS Y DESAFÍOS}

\author{
Marli Lopes Siqueira ${ }^{1}$, Suele Oliveira Cunha Costa $^{2}$, Anderson Reis de Sousa ${ }^{3}$, Delmo de \\ Carvalho Alencar ${ }^{4}$, Alyne Leal de Alencar Luz ${ }^{5}$, Álvaro Pereira ${ }^{6}$
}

Como citar esse artigo: Siqueira ML, Costa SOC, Sousa AR, Alencar DC, Luz ALA, Pereira A. Consulta de enfermagem à saúde de homens na atenção primária à saúde: estratégias e desafios. Rev Enferm Atenção Saúde [Internet]. 2021 [acesso em__];10(2):e202123. doi:10.18554/reas.v10i2.4245

\section{RESUMO}

Objetivo: Conhecer a efetividade da consulta de enfermagem à saúde de homens na Atenção Primária à Saúde em uma cidade do nordeste brasileiro. Método: Estudo exploratório, com abordagem qualitativa, realizado com enfermeiras da Atenção Primária à Saúde, no período de julho e agosto de 2015. Os dados foram submetidos ao método do Discurso do Sujeito Coletivo. Resultados: Evidenciou-se ausência de agenda específica para saúde de homens e baixa procura masculina pelo atendimento de Enfermagem, com baixa valorização da atuação da enfermeira. A Consulta de Enfermagem está planejada a partir de programas da Atenção Primária à Saúde, com demanda espontânea, estruturada com ênfase na queixa e no tratamento de doenças, permeados por entraves oriundos das masculinidades hegemônicas. Conclusão: Percebeu-se dificuldades das enfermeiras em abranger a população masculina nas ações de promoção à saúde, se revelando como uma prática incipiente e fragmentada, permeada por muitas dificuldades para ser desenvolvida a contento.

Descritores: Enfermagem; Processo de Enfermagem; Saúde do Homem; Atenção Primária à Saúde.

\footnotetext{
${ }^{1}$ Graduação em Enfermagem pela Faculdade Nobre de Feira de Santana (FAN), Feira de Santana, Bahia, Brasil. Faculdade Nobre (FAN), Feira de Santana-Bahia. http://orcid.org/0000-0003-1855-0302

${ }^{2}$ Graduação em Enfermagem pela Faculdade Nobre de Feira de Santana (FAN), Feira de Santana, Bahia, Brasil. Faculdade Nobre (FAN), Feira de Santana-Bahia. http://orcid.org/0000-0002-2797-5567

${ }^{3}$ Doutorando em Enfermagem e Saúde pelo Programa de Pós-Graduação em Enfermagem e Saúde da Escola de Enfermagem da Universidade Federal da Bahia (UFBA). Salvador, Bahia, Brasil. Escola de Enfermagem da Universidade Federal da Bahia (EEUFBA), Salvador-Bahia. http://orcid.org/0000-0001-8534-1960

4 Doutorando em Saúde Pública pela Escola Nacional de Saúde Pública da Fundação Oswaldo Cruz (ENSP/FIOCRUZ). Rio de Janeiro, Brasil. Escola Nacional de Saúde Pública. Fundação Oswaldo Cruz (ENSP/FIOCRUZ), Rio de Janeiro-RJ. http://orcid.org/0000-0002-6555-7921

${ }^{5}$ Doutoranda em Epidemiologia em Saúde Pública pela Escola Nacional de Saúde Pública da Fundação Oswaldo Cruz (ENSP/FIOCRUZ). Rio de Janeiro, Brasil. Escola Nacional de Saúde Pública. Fundação Oswaldo Cruz (ENSP/FIOCRUZ), Rio de Janeiro-RJ. http://orcid.org/0000-0003-1414-1026

${ }^{6}$ Doutor em Filosofia da Enfermagem pela Universidade Federal de Santa Catarina (UFSC). Professor da Escola de Enfermagem da Universidade Federal da Bahia. Salvador, Bahia, Brasil. Escola de Enfermagem da Universidade Federal da Bahia (EEUFBA), Salvador-Bahia. http://orcid.org/0000-0003-1615-5528
} 


\begin{abstract}
Objective: To learn about the effectiveness of nursing consultations for men's health in Primary Health Care in a city in northeastern Brazil. Method: Exploratory study, with a qualitative approach, carried out with Primary Health Care nurses, in the period of July and August 2015. The data were submitted to the Collective Subject Discourse method. Results: There was an absence of a specific agenda for men's health and low male demand for nursing care, with low appreciation for the nurse's performance. The Nursing Consultation is planned based on Primary Health Care programs, with spontaneous demand, structured with an emphasis on complaints and treatment of diseases, permeated by obstacles arising from hegemonic masculinities. Conclusion: Nurses' difficulties were perceived in reaching the male population in health promotion actions, revealing themselves as an incipient and fragmented practice, permeated by many difficulties to be successfully developed.
\end{abstract}

Keywords: Nursing; Nursing Process; Men’s Health; Primary Health Care.

\title{
RESUMEN
}

Objetivo: Conocer la efectividad de las consultas de enfermería para la salud del hombre en la Atención Primaria de la Salud en una ciudad del noreste de Brasil. Método: Estudio exploratorio, con enfoque cualitativo, realizado con enfermeras de Atención Primaria de la Salud, en julio y agosto de 2015. Los datos se sometieron al método de Discurso del Sujeto Colectivo. Resultados: No había una agenda específica para la salud de los hombres, la demanda masculina de atención de enfermería era baja y le daban poco valor al trabajo de la enfermera. La Consulta de Enfermería se planifica a partir de los programas de la Atención Primaria de la Salud, según la demanda espontánea, está estructurada en función de la queja y el tratamiento de enfermedades, permeados por obstáculos derivados de la masculinidad hegemónica. Conclusión: Se observó que las enfermeras tenían dificultades para incluir a la población masculina en las acciones de promoción de la salud, lo que revela que dicha práctica es incipiente y fragmentada, y cuenta con muchas dificultades para ser llevada a cabo satisfactoriamente.

Descriptores: Enfermería; Proceso de Enfermería; Salud del Hombre; Atención Primaria de la Salud.

\section{INTRODUÇÃO}

A literatura tem afirmado que os homens são mais vulneráveis às doenças, sobretudo às enfermidades graves $\mathrm{e}$ crônicas, o que leva à expectativa de vida, em média, sete anos mais baixa que a das mulheres. A maior vulnerabilidade e as altas taxas de morbimortalidade se justificam, em parte, pelo fato de os homens não buscarem os serviços de atenção primária, o que tem como consequência o agravo da morbidade e o retardamento na assistência. ${ }^{1}$
De acordo com o Ministério da Saúde $^{1}$, os elementos culturais de estereótipo e gênero masculino são os principais motivos da não adesão dos homens aos serviços de atenção primária, prestados nas unidades básicas de saúde. Esse fenômeno se constitui com raízes implantadas há séculos na sociedade, em que o gênero masculino foi constituído como referência de força, trabalho e desenvolvimento da sociedade. Nessa perspectiva, a doença é vista entre essa população como um sinal de fraqueza e 
fragilidade e não é devidamente reconhecida pelos homens como inerente à sua própria condição biológica. ${ }^{2}$

Portanto, com a finalidade de promover ações de saúde que contribuam significativamente para as demandas da população masculina, foi instituída, em 2008, a Política Nacional de Atenção Integral à Saúde do Homem. Tal política passa a reconhecer que os agravos do sexo masculino também constituem verdadeiros problemas de saúde pública, passando assim a ser inserido um novo contexto de atuação para o sistema de saúde então vigente, cujos eixos versam basicamente, sobre: violência, tendência à exposição a riscos com consequência nos indicadores de morbimortalidade, saúde sexual e reprodutiva, com ênfase nas questões de prevenção. ${ }^{3}$

Porém, o desafio de conseguir a adesão do público masculino aos programas de saúde é lançado, considerando-se a complexa singularidade que envolve o homem em seu perfil de gênero e estereótipo e ponderando as procedências do papel social em que o homem está inserido. Além disso, a escassez de programas voltados para a saúde masculina quase inviabiliza o atendimento especializado ao homem, levando esses indivíduos a reprimirem suas necessidades, procurando menos os serviços que as mulheres. Sendo assim a Enfermagem precisa traçar planos de cuidados ao homem que ofereça suporte informativo com relação aos cuidados gerais ligados à saúde. $^{4}$

A enfermeira, no contexto da Atenção Básica à Saúde, tem atribuições específicas dentre outras, a realização da Consulta de Enfermagem $(\mathrm{CE}){ }^{5}$ A realização da $\mathrm{CE}$ tem seu arcabouço legal sustentado pela Lei do Exercício Profissional, $N^{\circ} 7.498 / 86$, que a legitima como sendo uma atividade privativa do enfermeiro. Além disso, encontra-se ancorada pela Resolução 544 de 2017, em revogação à Resolução 159 de 1993, que dispõe especificamente sobre a CE. ${ }^{6}$

A realização da $\mathrm{CE}$ envolve tomada de decisão baseada em conhecimentos científicos e procedimentos que são sistematizados e avaliados constantemente, tornando-se um importante instrumento para o desenvolvimento do pensamento clínico necessário para o cuidado de enfermagem. ${ }^{7}$

Nesta perspectiva, este estudo tem o objetivo de conhecer a efetividade da Consulta de Enfermagem à saúde de homens na Atenção Primária à Saúde em uma cidade do nordeste brasileiro.

\section{MÉTODO}

Estudo exploratório, qualitativo, realizado com 22 enfermeiras que atuam em 15 Unidades de Saúde da Família (USF), da 
Atenção Primária do município de Feira de Santana, Bahia, Brasil.

A coleta de dados foi realizada durante os meses de julho e agosto de 2015. Adotou-se como critério de inclusão: profissionais enfermeiras (os) que exerciam o cargo há pelo menos um ano. Os de exclusão foram: profissionais que atuavam na gestão e administração dos serviços, sem contato direto com a assistência aos usuários ou que estavam ausentes das atividades laborais por motivos de férias ou afastamentos.

Utilizou-se como técnica para coleta de dados, uma entrevista individual, com tempo médio de 40 minutos de duração, guiada sob um roteiro semiestruturado, que subsidiou a caracterização sociodemográfica e profissional dos participantes. As enfermeiras foram convidadas a participarem do estudo, sendo orientadas sobre a finalidade, conferindo-as a oportunidade de desistirem a qualquer momento, sem que houvesse qualquer prejuízo.

As entrevistas foram gravadas, transcritas na íntegra e organizadas. Para tal estratégia, como ferramenta de apoio, atendeu-se aos critérios consolidados para o Consolidated Criteria for Reporting Qualitative Research (COREQ).

Para o tratamento do material empírico foi realizada sistematização através do Software NVIVO®, e adotado o Rev Enferm Atenção Saúde [Online]. Jul/Set 2021; 10(2):e202123 método de organização e apresentação dos dados, baseando-se no Discurso do Sujeito Coletivo (DSC), que permitiu gerar as figuras metodológicas: Expressões Chaves, Ideias Centrais, Ancoragens e o DSC..$^{8-9}$ Tal método, fundamenta-se na Teoria das Representações Sociais e de pressupostos sociológicos. ${ }^{9}$ A análise é ancorada a partir do referencial normativo de Consulta de Enfermagem, disposto na Resolução 544 de 2017, em revogação à Resolução 159 de 1993 do Conselho Federal de Enfermagem. ${ }^{6}$

O estudo foi aprovado pelo Comitê de Ética em Pesquisa da Faculdade Nobre (FAN), sob Parecer $n^{\circ} 1.241 .569 / 2015$. As participantes assinaram o Termo de Consentimento Livre e Esclarecido (TCLE). Todas as etapas do estudo foram fundamentadas na Resolução nº 466/12 do Conselho Nacional de Saúde.

\section{RESULTADOS}

IDEIA CENTRAL SÍNTESE 1: PLANEJAMENTO, ORGANIZAÇÃO E ESTRUTURAÇÃO DA CONSULTA DE ENFERMAGEM

\section{Ideia Central Síntese 1A: Ausência de agenda específica e baixa procura masculina}

Em minha atuação são realizadas consultas para homens, as mesmas são agendadas semanalmente no Planejamento Familiar, *HIPERDIA e Pré-natal, sendo estas realizadas por demanda espontânea, baseada na queixa trazida por eles. Não existe na unidade um turno específico para atendimento ao 
homem, já até tentamos abrir, mas fica complicado deixar uma agenda de Enfermagem aberta só para atender os homens, pois a demanda é baixa. A consulta que é realizada é marcada mediante ao atendimento de acordo com os programas já existentes na unidade. Não agendamos dias específicos para eles, deixando por livre demanda, $e$ não designo dias para consulta para eles, porque não temos sucesso na procura, eles não vêm e quando eles aparecem, já é para tratar algum problema. Mas, se eles aparecerem, eu acolho, e não deixo de atender, justamente por ser um público de difícil procura. Os homens que aparecem mais às consultas de Enfermagem, são aqueles que já têm alguma patologia crônica, como a Hipertensão Arterial e a Diabetes Mellitus, estes, digamos que já são os pacientes certos de realizarem a consulta. (DSC de enfermeiras que atuam na APS).

*HIPERDIA - Programa de Prevenção $e$ Tratamento da Hipertensão e Diabetes do Ministério da Saúde, Brasil.

\section{Ideia Central Síntese 1B: Desvalorização da Consulta de Enfermagem}

O homem vem mais para consulta médica, eles não valorizam muito meu atendimento enquanto enfermeira, até porque eles querem curar alguma coisa, e nós não passamos nenhum medicamento, só os da cobertura ou se houver assinatura do médico, então, acredito eu, que eles acham melhor ir logo ao médico do que ir para a consulta com a enfermeira. O que me parece é que eles não confiam na atuação de uma enfermeira, não sei se por vergonha, ou por outro motivo. Isso também se torna mais perceptível quando os Agentes Comunitários de Saúde me trazem as demandas, que são maiores para o médico clínico, do que para a enfermeira. Para solucionar essa situação, temos tentado desenvolver a estratégia de atuar junto com os Agentes
Comunitários de Saúde, para poderem resgatá-los. Temos um cronograma de atividades mensais $e$ semanais que é dado pela Secretaria Municipal de Saúde, onde seguimos à risca. Para tanto, procuro promover a aproximação com esse público, realizando sala de espera, ações em saúde, atividades educativas, feiras de saúde, como forma de poder atrair este público e poder cumprir com cronograma solicitado. (DSC de enfermeiras que atuam na APS).

\section{IDEIA CENTRAL SÍNTESE 2: OPERACIONALIZAÇÃO DA CONSULTA DE ENFERMAGEM}

\section{Ideia Central Síntese 2A: Estrutura e direcionamento da Consulta de Enfermagem}

Inicialmente, acolho os homens e abordo de maneira geral, o vejo como um todo, tento encontrar suas necessidades e pergunto sobre sua patologia, o que está sentindo. Procuro saber se tem histórico familiar de alguma doença crônica e oriento para sempre comparecer às consultas. Minha abordagem é sucinta e simples, porque se você questionar muito eles se travam, não se soltam e não vêm mais. Avalio idade, patologia, uso de medicamentos, frequência de exames laboratoriais, hidratação, alimentação, hábitos saudáveis, higiene, se fuma, se bebe, condições de vida, atividade física e riscos à saúde. Após saber essas questões, tiro dúvidas, procuro sempre saber como e quando eles usam suas medicações, faço destaque para a importância da ingesta hídrica, da alimentação equilibrada, do uso correto dos medicamentos, explico a importância do exame da próstata, independente da faixa etária, pois, todos esses homens podem ser multiplicadores de informações a respeito deste tipo de exame, uma vez que pode existir alguém na família que não fez e precisa fazer o exame, elou conhecidos que seguem 
a linha de raciocínio masculino, criam resistência a tal procedimento, e assim poder ter uma boa qualidade de vida. Realizo avaliação e aferição de pressão arterial, glicemia. Procuro conversar com ele para solucionar os problemas, inclusive temos em nossa unidade, grupos de saúde do homem, que sempre estão realizando salas de espera com temas relevantes, tais como Infecções Sexualmente Transmissíveis, Planejamento Familiar, vasectomia, dentre outros temas. Como consequência dessas ações, a procura aumentou para realização de vasectomia. No que está sob o nosso olhar clínico, tentamos resolver, se não, encaminhamos para algum médico ou clínico, nutricionista, até mesmo dentista e encaminhamento para a sala de vacina, porque a maioria dos homens não tem cartão de vacina atualizado. (DSC de enfermeiras que atuam na $A P S)$.

\section{Ideia Central Síntese 2B: Entraves gerados pela construção hegemônica das masculinidades}

A saúde do homem é muito complicada, tem sido um desafio constante. A população do sexo masculino é resistente e não tem uma frequência constante na unidade de saúde, nem nos programas, sendo necessário muitas vezes realizar busca ativa nos domicílios, juntamente com os Agentes de Saúde. A Consulta de Enfermagem com os homens é mais difícil, pois eu tenho que saber conversar com eles, porque senão, com qualquer motivo eles podem não gostar e já ser um motivo para a ausência. Às vezes estou em atendimento e quando percebo eles já foram embora, não esperam. Os homens querem ser medicados, não tem paciência para aguardar pelo atendimento e não seguem as medidas repassadas durante a Consulta de Enfermagem, e por conta disso, a consulta acaba sendo breve e mais focada nas queixas apresentadas por eles, como forma de solucionar com maior rapidez os problemas. Buscar pela prevenção tem sido muito difícil por parte dos homens, aparecendo mais em contextos de reabilitação da saúde. Observo essa realidade, quando fazemos os grupos e as atividades para ver se eles aderem ao serviço, mas eles não aparecem, ou mesmo quando geram remarcações nos atendimentos devido as ausências ou desistências. Muitas [as esposas] dizem que seus maridos não têm tempo para virem até a unidade. Até para assinarem a ata de ligadura, no caso da contracepção, os homens tem enfrentado dificuldade, porque não têm tempo para virem até o serviço devido a limitação de horário. Durante as consultas de planejamento familiar, o desafio tem sido os homens acompanharem as mulheres. A adesão acaba sendo daqueles com doenças crônicas ou patologias de base, em idade avançada ou em situação de urgência e emergência. (DSC de enfermeiras que atuam na APS).

\section{Ideia Central Síntese 2B: Dificuldades para aplicação da Consulta de Enfermagem}

Tenho dificuldade em realizar o atendimento direcionado aos homens, pois eles não comparecem na consulta, pois muitas vezes estão no trabalho. Tento rastrear, mas se torna difícil, pois não conheço a comunidade, e acabo me limitando às informações repassadas pelo Agente Comunitário de Saúde e é com base nessas informações que eu tento agendar uma visita no domicílio para ir atrás dos homens. (DSC de enfermeiras que atuam na $A P S)$.

\section{Ideia Central Síntese 2C: Estratégias para realizar a Consulta de Enfermagem}

Estratégia específica não tem, aproveito a presença na consulta de pré-natal ou planejamento familiar e reforço a importância da vinda deles às 
consultas, e quando eles não vêm, aproveito as esposas de forma direta para levar a guia de exames e convencer eles a virem ao serviço, ou então aproveito a presença deles e já direciono cuidados e acolhimento aos mesmos. Eles aparecem mais na campanha do novembro azul e estão procurando mais por causa do PSA, caso tenham alteração no exame eles fazem o toque, mas se fosse para fazer o toque especificamente, eles ainda têm uma certa resistência e preconceito e não fariam, acredito que seja a questão de gênero. Considero como estratégia os panfletos que a secretaria de saúde disponibiliza, que são específicos para os homens e a comunicação com os ACS, de forma indireta, e sempre que tiver necessidade, fazemos a busca ativa daqueles pacientes faltosos. (DSC de enfermeiras que atuam na APS).

\section{DISCUSSÃO}

Socialmente, é imposta aos homens uma postura que denota invulnerabilidade e o cultivo da ideia utópica de que os homens não adoecem, já que a doença é um sinal de fragilidade. Tal postura advém da educação dada às crianças que enfatiza a oposição dos gêneros, de modo que o padrão masculino busca a afirmação da virilidade a partir do distanciamento de comportamentos ditos femininos, estruturando uma construção hegemônica das masculinidades. ${ }^{3}$

Compreende-se então que, os homens buscam os serviços de saúde quando há uma condição aguda que compromete sua saúde, não compreendendo a importância das ações de promoção da saúde, já que a ida aos serviços de saúde é entendida como um sinal de fragilidade e um comportamento tipicamente feminino. ${ }^{10}$ Essa construção é histórica, pois baseia-se no modelo ainda hegemônico vigente, que se transpõe para a dinâmica de cuidado no sistema de saúde, que não reconhece, muitas vezes, as necessidades demandadas pelo público masculino, nem oportuniza possibilidades de ampliação do acesso de homens aos serviços, fazendo com que estes não sejam reconhecidos enquanto sujeitos de cuidados. ${ }^{11}$

Além disso, o processo de construção hegemônico das masculinidades, repercute na não estimulação dos homens em prol do exercício do autocuidado e do estabelecimento de uma cultura de cuidado com a saúde, bem como do desenvolvimento de uma rotina de cuidados junto aos serviços e aos profissionais de saúde, que se expressam no pouco pertencimento dos homens nesse cotidiano, devendo assim, ser melhor compreendidos. ${ }^{11}$

Além da cultura que valoriza o comportamento pautado na oposição de gênero, existem outras razões para a ausência dos homens nas unidades de saúde, como a falta de sistematização da assistência e a precarização do atendimento no serviço público. Os homens priorizam as atividades laborais, em detrimento do cuidado à saúde, de modo que para eles, a ideia de ir para um serviço de saúde 
significa a perda do tempo que seria destinado ao trabalho. ${ }^{12}$

Entretanto, há que se considerar que o trabalho constitui uma dimensão relevante na condição humana, e se estabelece enquanto uma forma de cuidado, que não necessariamente, se restringe à ida a um serviço de saúde, sendo, portanto, necessário repensar sobre a criação de novas estratégias de inserção masculina nos serviços de APS, como por exemplo, na extensão de horários, turnos e dias de atendimento.

O discurso revelou que o programa de saúde HIPERDIA, voltado para os usuários com Hipertensão Arterial e Diabetes Mellitus, configura-se como um dos grandes atrativos que leva os homens às unidades de saúde na Atenção Primária, em especial os de idade avançada, não sendo contemplado como foco de atenção e interesse por parte das enfermeiras, os homens adolescentes e adultos jovens. Tal constatação é preocupante, uma vez que são os homens jovens e adultos entre 20-29 anos que conformam entre as estatísticas de mortalidade, principalmente por causas externas, doenças cardiovasculares, respiratórias e digestivas. ${ }^{13}$

No cenário investigado, verificou-se grande procura masculina por terapêutica medicamentosa e/ou transcrição da prescrição médica realizada por parte das enfermeiras, mostrando-se uma barreira no processo de trabalho em Enfermagem, o que dificulta a inserção dos homens em outros programas, tais como, planejamento familiar e reprodutivo, ações de promoção da paternidade presente e responsável, assistência pré-natal, crescimento e desenvolvimento da criança, atividades coletivas, práticas corporais, atenção à saúde bucal, imunização, dentre outras. Isso implica ainda, na não compreensão dos homens acerca da relevância e da essencialidade do trabalho da enfermeira na APS, enfraquecendo a visibilidade das dimensões do trabalho em Enfermagem.

Culturalmente os homens buscam os serviços de saúde quando já existe uma afecção manifestada, priorizando o atendimento curativo. Por conta de tal comportamento, eles objetivam o atendimento médico nas unidades de saúde, para rapidamente ter a receita médica que resolva sua alteração orgânica. As atividades de promoção da saúde e prevenção de agravos são atribuídas, pela população masculina, às necessidades femininas, dispensando tais orientações, que normalmente são dadas pela Enfermagem. ${ }^{3,5,7,12}$

Dado este cenário, importa pensar na potencialização do trabalho colaborativo e interprofissional em saúde, a partir da criação de atividades conjuntas, como forma de fortalecer o trabalho em equipe na Estratégia Saúde da Família, quer seja, por 
meio do atendimento coletivo, da operacionalização dos projetos terapêuticos singulares, do matriciamento, da criação de agendas conjuntas de saúde, dentre outras, bem como, aumentar a demanda de homens nos serviços de saúde e o fortalecimento da protagonização da atuação da enfermeira.

Outro ponto importante a ser considerado, a partir dos relatos, é a iniciativa incipiente ou ausente da enfermeira para o desenvolvimento de atividades que transponham as normativas do governo federal ou da secretaria municipal de saúde. Percebeu-se que as enfermeiras se preocupam em cumprir o que lhes é solicitado, sentindo-se enfadada quando há novos impressos, novas metas a serem cumpridas, não desvelando preocupação com a garantia da atenção integral à saúde dos homens e o desenvolvimento da produção de um cuidado específico. Neste sentido, a essencialidade do trabalho da enfermeira na atenção à saúde de homens, deve ir além da dimensão técnica, restritiva ao cumprimento de normas, rotinas e cronogramas, para pautar o cuidado enquanto uma prática social, da qual compreende o homem, a sua família e as suas inserções na sociedade.

As enfermeiras (os) devem fortalecer a execução da $\mathrm{CE}$, uma vez que a sua institucionalização, configura-se num processo da prática de Enfermagem, e se concretiza em um modelo assistencial adequado e compatível com as necessidades de saúde da população. ${ }^{6}$ A sua intervenção é de baixo custo e de simples aplicação, todavia, o seu desenvolvimento é envolvido de habilidades de ordem cognitiva, interpessoal e psicomotora, assim como de pensamento crítico e expertise clínica. ${ }^{14}$

A culpabilização dos homens para a ausência aos serviços de saúde, deve ser uma postura abandonada pela equipe de saúde, uma vez que a enfermeira deve conhecer a realidade das famílias adscritas em sua área, para um adequado planejamento e desenvolvimento de ações individuais e coletivas. Identificado o perfil da população masculina, a enfermeira dispõe de ferramentas, como a visita domiciliar e a educação em saúde, para chegar até aqueles homens que dificilmente iriam à unidade, de modo espontâneo, além de promover uma abordagem participativa que permita que o usuário participe de modo protagonista no processo. ${ }^{15}$

A operacionalização da CE não apresenta uma sistematização de consulta, mostra-se carente de subsídios e pressupostos teóricos ordenadores e orientadores para a sua execução no campo de prática, sendo guiada a partir da operacionalização de programas de saúde, já instituídos pelo Ministério de Saúde brasileiro. Não se percebeu no discurso, a construção de um cuidado de Enfermagem 
com especificidade às demandas de saúde masculina, mesmo considerando a necessidade de garantir atenção especializada às necessidades de saúde, a partir do levantamento de problemas, diagnósticos, metas e resultados específicos de Enfermagem. A não incorporação desses preceitos, pode implicar na não adesão desse público às $\mathrm{CE}$, dado o baixo reconhecimento e pertencimento dos homens ao cuidado produzido por enfermeiras na APS. Diante dessa problemática, cabe aos serviços de saúde sensibilizar os homens, para as ações de promoção da saúde, realizando busca ativa, caso seja necessário. ${ }^{4,16,17}$

Além dessa dificuldade enfrentada no acesso à população masculina, enfatiza-se a necessidade de educação permanente de recursos humanos em saúde, para atuarem frente à saúde de homens. Neste sentido, considerando que a CE tem como fundamento os princípios de universalidade, equidade, resolutividade e integralidade das ações de saúde, a formação em saúde se mostra essencial para que esses princípios sejam alcançados na prática. ${ }^{18}$

\section{CONCLUSÃO}

O estudo demonstrou baixa procura masculina pelo atendimento de Enfermagem, com baixa valorização da atuação da enfermeira e da produção do cuidado de Enfermagem por parte dos usuários, o que repercute no desenvolvimento e avanço da Assistência de Enfermagem.

O contexto de aplicação da CE está permeado por entraves oriundos da contextualização social hegemônica das masculinidades, que interferem na relação de proximidade dos homens com o contexto de cuidado à saúde promovido nos serviços, tal como, da dificuldade enfrentada pelas enfermeiras no acesso ao público masculino no território em que atuam. As estratégias utilizadas a fim de solucionar o problema, parecem não obter sucesso, dado o contexto de distanciamento dos homens das estratégias promovidas nos serviços.

O estudo apresentou como limitações: a dificuldade de acesso às enfermeiras para realização das entrevistas, escassez de instrumentalização destas, no tocante à produção do cuidado à saúde de homens e a ausência de fundamentos teóricos da consulta, com direcionamentos para as demandas de saúde do público masculino.

\section{REFERÊNCIAS}

1. Ministério da Saúde (Brasil). Política nacional de atenção integral à saúde do homem [Internet]. Brasília, DF: Ministério da Saúde; 2008 [citado em 22 set 2021]. Disponível em: https://bvsms.saude.gov.br/bvs/publicacoes /politica_nacional_atencao_homem.pdf 2. Pereira J, Klein C, Meyer DE. PNAISH: uma análise de sua dimensão educativa na perspectiva de gênero. Saúde Soc. [Internet]. 2019 [citado em 12 dez 2019]; 
28(2):132-46. Disponível em:

https://www.scielo.br/scielo.php?script=sci _arttext\&pid=S0104-

$12902019000200011 \& \operatorname{lng}=$ pt\&nrm=iso\&tl $\mathrm{ng}=\mathrm{pt}$

3. Alves BMS, Araújo CJS, Almeida SLS, Guimarães ALS. Atuação do enfermeiro da atenção básica diante das dificuldades para a implementação da política de saúde do homem. Rev Enferm UFPE online [Internet]. 2017 [citado em 24 out 2019]; 11(Supl.12):5391-401. Disponível em: https://periodicos.ufpe.br/revistas/revistaen fermagem/article/view/110143

4. Arruda GO, Marcon SS. Reflexões sobre o cuidado ao homem adulto doente no âmbito familiar: uma perspectiva de gênero. Rev Enferm UFSM. [Internet]. 2016 [citado em 11 nov 2019]; 6(2):298306. Disponível em:

https://periodicos.ufsm.br/reufsm/article/vi ew/19344

5. Silva KM, Santos SMA. A consulta de enfermagem na estratégia de saúde da família: realidade de um distrito sanitário. Rev Enferm UFSM. [Internet]. 2016 [citado em 24 out 2019]; 6(2):248-58. Disponível em:

https://periodicos.ufsm.br/reufsm/article/vi ew/18079

6. Conselho Federal de Enfermagem (Brasil). Resolução COFEN no 544, de 09 de maio de 2017. Revoga a Resolução $\mathrm{n}^{\circ}$ 159/1993, que dispõe sobre a Consulta de Enfermagem. Brasília, DF: COFEN; 2017. Disponível em:

http://www.cofen.gov.br/resolucao-cofenno-05442017_52029.html

7. Teston EF, Peternella FMN, Sales CA, Haddad MCL, Cubas MR, Marcon SS. Efeito da consulta de enfermagem no conhecimento, qualidade de vida, atitude frente à doença e autocuidado em pessoas com diabetes. REME Rev Min Enferm. [Internet]. 2018 [citado em 01 nov 2019]; 22:e-1106. Disponível em: http://www.reme.org.br/artigo/detalhes/124 2

8. N Vivo 10 for Windows. [Burlington, MA]: QSR International; 2014. Disponível em:

http://download.qsrinternational.com/Docu ment/NVivo10/NVivo10-Getting-Started-

Guide-Portuguese.pdf

9. Lefevre AMC, Crestana MF, Cornetta

VK. A utilização da metodologia do discurso do sujeito coletivo na avaliação qualitativa dos cursos de especialização "Capacitação e Desenvolvimento de Recursos Humanos em Saúde CADRHU”, São Paulo - 2002. Saúde Soc. [Internet]. 2003 [citado em 12 out 2019]; 12(2):68-75. Disponível em: https://www.scielo.br/scielo.php?pid=S010 $4-$

$12902003000200007 \&$ script=sci_abstract \&tlng $=\mathrm{pt}$

10. Arruda GO, Corrêa ACP, Marcon SS.

Fatores associados aos indicadores de necessidades em saúde de homens adultos. Acta Paul Enferm. [Internet]. 2014 [citado em 22 nov 2019]; 27(6):560-6. Disponível em:

https://www.scielo.br/pdf/ape/v27n6/19820194-ape-027-006-0560.pdf

11. Moura EC, Santos W, Neves ACM,

Gomes R, Schwars E. Atenção à saúde dos homens no âmbito da Estratégia Saúde da Família. Ciênc Saúde Colet. [Internet]. 2014 [citado em 22 nov 2019]; 19(2):42938. Disponível em: https://www.scielo.br/scielo.php?pid=S141 $3-$

$81232014000200429 \&$ script=sci_abstract \&tlng=pt

12. Bidinotto DNPB, Simonetti JP, Bocchi

SCM. A saúde do homem: doenças crônicas não transmissíveis e vulnerabilidade social. Rev Latinoam Enferm. [Internet]. 2016 [citado em $11 \mathrm{dez}$ 2019]; 24:e2756. Disponível em: https://www.scielo.br/pdf/rlae/v24/pt_0104 -1169-rlae-24-02756.pdf

13. Pereira LP, Nery AA. Planejamento, gestão e ações à saúde do homem na estratégia de saúde da família. Esc Anna Nery [Internet]. 2014 [citado em $11 \mathrm{dez}$ 2019]; 18(4):635-43. Disponível em: https://www.scielo.br/scielo.php?pid=S141 $4-$ 
$81452014000400635 \&$ script=sci_abstract \&tlng $=\mathrm{pt}$

14. Imazu MFM, Faria BN, Arruda GO, Sales CA, Marcon SS. Effectiveness of individual and group interventions for people with type 2 diabetes. Rev Latinoam Enferm. [Internet]. 2015 [citado em 15 nov 2019]; 23(2):200-7. Disponível em:

https://www.scielo.br/scielo.php?script=sci _arttext\&pid=S0104-11692015000200004 15. Teixeira DBS, Cruz SPL. Atenção à saúde do homem: análise da sua resistência na procura dos serviços de saúde. Rev Cubana Enferm. [Internet]. 2016 [citado em 22 nov 2019]; 32(4):126-36.

Disponível em:

http://scielo.sld.cu/pdf/enf/v32n4/enf11416 .pdf 16. Barros CT, Gontijo DT, Lyra J, Lima LS, Monteiro EMLM. "Mas se o homem cuidar da saúde fica meio que paradoxal ao trabalho": relação entre masculinidades e cuidado à saúde para homens jovens em formação profissional. Saúde Soc.

[Internet]. 2018 [citado em 15 out 2019]; 27(2):423-34. Disponível em:

https://www.scielo.br/pdf/sausoc/v27n2/19 84-0470-sausoc-27-02-423.pdf

17. Silva TFA, Rodrigues JEG, Silva PSM, Barros MAR, Felipe GF, Machado ALG.

Nursing consultation to persons with diabetes mellitus in primary care. REME Rev Min Enferm. [Internet]. 2014 [citado em 01 dez 2019]; 18(3):710-6. Disponível em:

https://cdn.publisher.gn1.link/reme.org.br/ pdf/en_v18n3a15.pdf

18. Silva AN, Silva SA, Silva ARV, Araújo TME, Rebouças CBA, Nogueira LT. A avaliação da atenção primária a saúde na perspectiva da população masculina. Rev Bras Enferm. [Internet]. 2018 [citado em $01 \mathrm{dez} 2019$ ]; 71(2):25563. Disponível em: https://www.scielo.br/pdf/reben/v71n2/pt 0034-7167-reben-71-02-0236.pdf

RECEBIDO: $19 / 01 / 20$

APROVADO: 03/03/21

PUBLICADO: 09/2021 